\title{
Supporting Tropos concepts in Agent OPEN ${ }^{\star}$
}

\author{
Brian Henderson-Sellers ${ }^{1}$, Paolo Giorgini ${ }^{2}$, and Paolo Bresciani ${ }^{3}$ \\ 1 University of Technology, Sydney, NSW 2007 Australia, \\ brian@it.uts. edu.au, \\ ${ }^{2}$ Department of Information and Communication Technology \\ University of Trento, Trento, Italy \\ paolo.giorgini@dit.unitn. it \\ 3 ITC-irst, Povo (Trento), Italy \\ bresciani@itc.it
}

\begin{abstract}
The growth of interest in agent-orientation as a new paradigm has introduced the need for developing concepts, tools and techniques for modeling and engineering agent-based software systems. Object technology has been supporting the development of information systems for many years but is now slowly evolving to encompass more recent ideas relating to the concept of "agent". Integrating agent concepts into existing OO methodologies has resulted in several agent-oriented methodologies, one of which is Agent OPEN. In this paper, we evaluate the existing Agent OPEN description against ideas formulated within Tropos, an agent-oriented software development methodology.
\end{abstract}

\section{Introduction}

The explosive growth of application areas such as electronic commerce, enterprise resource planning and mobile computing has profoundly and irreversibly changed our views on software and Software Engineering. Software must now be based on open architectures that continuously change and evolve to accommodate new components and meet new requirements. Software must also operate on different platforms, without recompilation, and with minimal assumptions about its operating environment and its users. In addition, software must be robust and autonomous, capable of serving a naïve user with a minimum of overhead and interference. These new requirements, in turn, call for new concepts, tools and techniques for engineering and managing software.

For these reasons - and more - agent-oriented software development is gaining popularity over traditional software development techniques. While object technology has been in widespread use for the development of information systems for many years, new ideas from the agent-oriented community are beginning to be addressed by extending existing OO methodologies to support the development of agent-based information systems (e.g., [27]). This is particularly evident in the discussions regarding whether agent orientation is a brand new paradigm

\footnotetext{
* This is Contribution Number $03 / 10$ of the Centre for Object Technology Applications
} and Research (COTAR) 


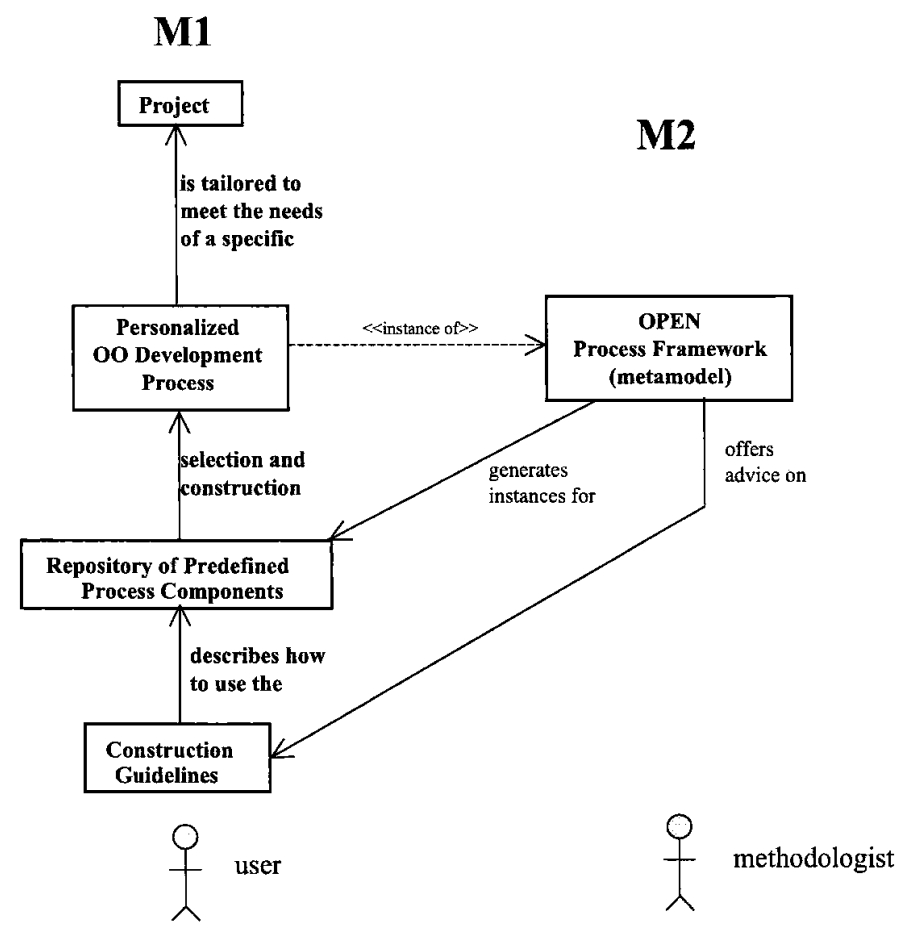

Fig. 1. The OPF metamodel generates a large number of instances from each metaclass, which are stored in a repository. Individual process components are then selected and used to construct the process.

requiring a non-OO mindset or whether it can be accommodated as an extension of existing $\mathrm{OO}$ ideas.

In this paper, we make the (common) assumption that adding support for agent concepts into an existing object-oriented methodological approach is feasible and useful. We begin with the OO approach offered by the OPEN Process Framework or OPF [9]. Although some basic agent concepts have recently been added [6] to create "Agent OPEN", Agent OPEN still lacks the sophisticated support for agents necessary to provide complete support for agent-oriented software development (AOSD).

Although it would be possible to use a combination of methodologies (e.g., here, OPEN complemented by Tropos), it is not practical for industry, which preferably requires a single, coherent and integrated "package" to support application development. This paper reports on the first results of a project intended to ensure that Agent OPEN's repository of process components contains complete support for AOSD. To accomplish this, each of the major AO methodologies 


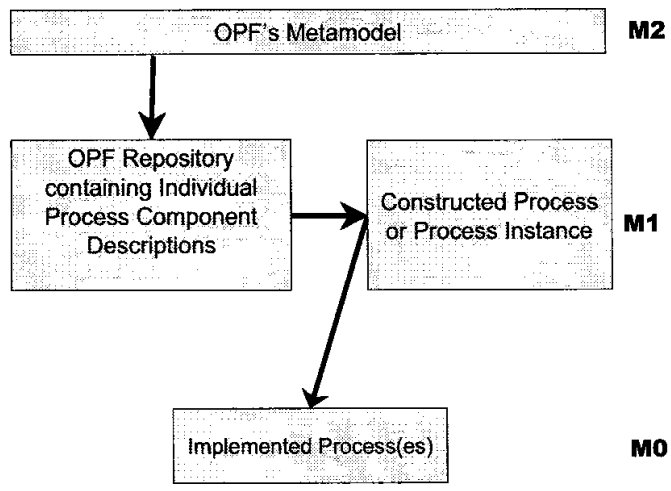

Fig. 2. Three metalevels (M2, M1, M0) that provide a framework in which the relationship between the metamodel (M2), the repository and process instances (M1) and the implemented process (M0) can be seen to co-exist.

is analyzed in turn in order to discover agent-oriented process components currently deficient in the OPF repository of process components - actual methodologies being constructed from these components (Figure 1) using the principles of method enginering [3]. The first AO methodology to be analyzed in this way is Tropos [2], which stresses the need for agent-orientation in early requirements engineering - topics not already addressed in the OPEN literature.

In Section 2, we present these premexisting agent extensions in the context of the OPF itself. In Section 3 we outline the Tropos methodology and then in Section 4 we evaluate whether the OPF in its extended form [6] is adequate to support the concepts and process elements described in Tropos and, where not, what further extensions are needed. We conclude in Section 5 with recommendations and outline directions for future work.

\section{The OPEN Process Framework and its Existing Agent-oriented Enhancements}

Integrating agent concepts into existing $\mathrm{OO}$ methodologies has resulted in several agent-oriented methodologies, for example, $[7,4,27,1]$. One which we will discuss is the OPEN Process Framework, or OPF $[11,17,9]$, which is a little different from most others in that it offers a metamodel-underpinned framework rather than (strictly) a methodology.

Method engineering (e.g., $[3,25]$ ) is then used to construct project-specific or "situational" methods (a.k.a. methodologies). This is possible because of the provision of a repository of method fragments (e.g., [26]) or process components (e.g., [9]). 


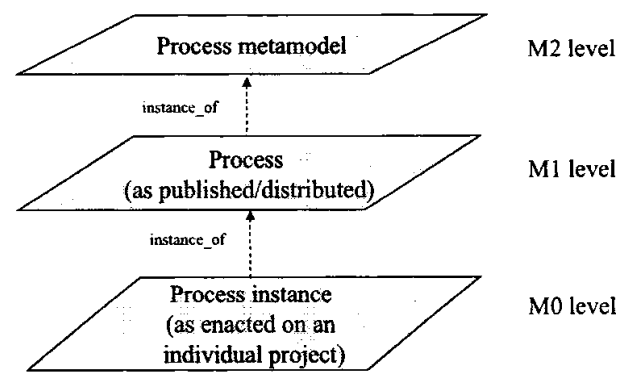

Fig. 3. For process metamodelling, we adopt a 3 layer version of the 4 layer OMG UML metamodel [19] i which every concept in layer $x$ is an instance of a concept at level $x+1$ (except for the self-referential top layer).

Initially, the repository of method fragments in OPEN was aimed at providing the ability to construct methodologies in the general area of information systems development. However, as new ideas emerged over the last few years, projects to extend the contents of the OPF repository have seen additions in areas such as component-based development [14], web-based development $[13,12]$ and organizational transition [16]. Initial extensions to agent-oriented development were formulated in $[6,15]$ and it is these extensions which we evaluate for completeness against the agent-oriented Tropos methodology - a comparison which is the focus of this paper.

\subsection{The OPEN Process Framework}

OPF consists of (i) a process metamodel or framework from which can be generated an organizationally-specific process (instance) created, using a method engineering approach [3], from (ii) a repository and (iii) a set of construction guidelines. The metamodel can be said to be at the M2 metalevel (Figure 2) with both the repository contents and the constructed process instance at the M1 level (Figure 3). The M0 level in Figure 2 represents the execution of the organization's (M1) process on a single project. Each (M1) process instance is created by choosing specific process components from the OPF Repository (Figure 1) and constructing (using the Construction Guidelines) a specific configuration - the "personalized OO development process". Then, using this method engineering approach, from this process metamodel we can generate an organizationallyspecific process (instance).

The major elements in the OPF metamodel are Work Units (Activities, Tasks and Techniques), Work Products and Producers [9] — see Figure 4. These three components interact; for example producers perform work units, work units maintain work products and producers produce work products. In addition to these three metatypes, there are two auxiliary ones (Stages and Languages), which interact as shown in Figure 4. 


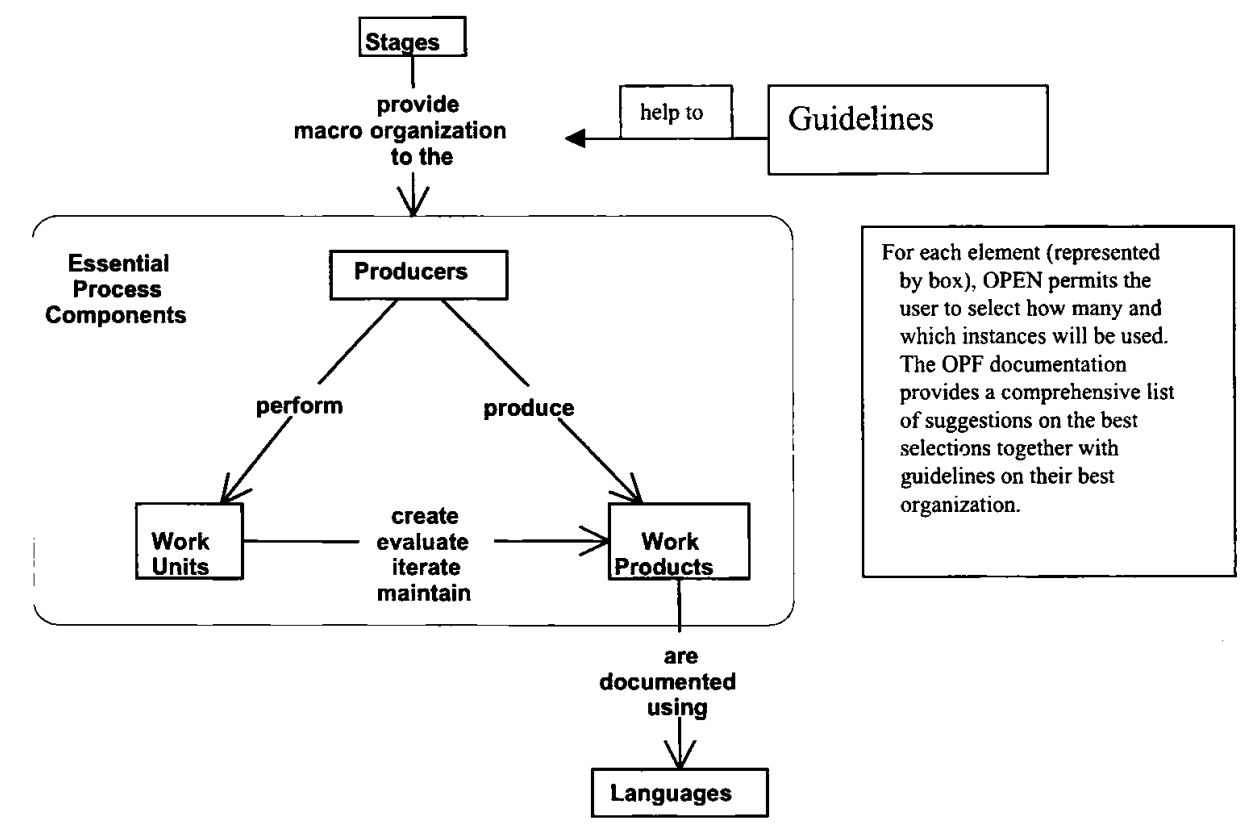

Fig. 4. The five major metatypes in the OPF metamodel (after [9]).

Activity is at the highest level in the sense that a process consists of a number of Activities. Activities are large scale definitions of what must be done. They are not used for project management or enactment because they are at too high an abstraction level. Instead, OPEN offers the concept of Task (in agreement with the terminology of the Project Managers' Body of Knowledge [8]) which is defined as being the smallest unit of work that can be project managed. Both Activities and Tasks are kinds of Work Unit in the OPF metamodel (Figure 4).

Work Products are the outputs of the Activities. These work products may be graphically or textually described. Thus, we need a variety of languages to describe them. Typical examples here are English (natural language), UML (modelling language) and $\mathrm{C \#} \mathrm{(implementation} \mathrm{language).} \mathrm{Since} \mathrm{the} \mathrm{metamodel} \mathrm{itself}$ is a "design model", it is reasonable to document it with one of the available modelling languages. Here, we use the Unified Modeling Language of the OMG [19] since it is probably the most commonly used (at least in $\mathrm{OO}$ developments).

While it is possible to analyze the metamodel directly, in this paper we address the issue of whether the contents of the current repository for the OPF is adequate for supporting agent-oriented developments. This repository contains instances generated from each of the metaclasses in the metamodel. For each metaclass there are potentially numerous instances. These are documented in 


\begin{tabular}{||l|l||}
\hline Tasks for AOIS & Techniques likely to be useful \\
\hline Identify agents' roles & Environmental evaluation \\
\hline Model the agent's environment & Environmental evaluation \\
\hline Identify system organization & Environmental evaluation \\
\hline $\begin{array}{l}\text { Determine agent interaction protocol } \\
\text { Determine delegation strategy }\end{array}$ & $\begin{array}{l}\text { Contract nets } \\
\text { Market mechanisms }\end{array}$ \\
\hline Determine agent communication protocol & FIPA KIF compliant language \\
\hline Determine conceptual architecture \\
Determine agent reasoning & $\begin{array}{l}\text { 3-layer BDI model } \\
\text { Deliberative reasoning: Plans } \\
\text { Reactive reasoning: ECA Rules }\end{array}$ \\
\hline Determine control architecture & $\begin{array}{l}\text { Belief revision of agents } \\
\text { Commitment management } \\
\text { Activity scheduling } \\
\text { Task selection by agents } \\
\text { Control architecture }\end{array}$ \\
\hline Determine system operation \\
Gather performance knowledge & Learning strategies for agents \\
\hline Determine security policy for agents & [topic of future research] \\
\hline Undertake agent personalization & $\begin{array}{l}\text { Environmental evaluation } \\
\text { User model incorporation }\end{array}$ \\
\hline Identify emergent behaviour & topic of future research] \\
\hline
\end{tabular}

Table 1. Tasks and Techniques already proposed $[6,15]$ for addition to the OPF repository in order to support the development of agent-oriented systems.

various books and papers, as noted earlier. The ones specific to agents are listed in Table 1 (see next section).

\subsection{The Current Agent OPEN}

As a consequence of the modular nature of the OPEN approach to methodology, via the notion of a repository of process components together with the application of method or process engineering [24], it is relatively easy to add additional meta-elements and extremely easy to add additional examples of process components to the repository (as instances of pre-existing meta-elements). To extend this approach to support agent-oriented information systems, Debenham and Henderson-Sellers [6] analyzed the differences between agent-oriented and object-oriented approaches in order to be able to itemize and outline the necessary additions to the OPEN Process Framework's repository in the standard format provided in [17]. The focus of that work was primarily on instances of the meta-class WorkUnit useful for agent-oriented methodologies and processes. Table 1 lists the Tasks and Techniques so far added to the OPF repository (no new Activities were identified). 


\section{The Tropos Methodology}

The Tropos methodology $[2,5,10,20]$ was designed to support agent-oriented systems development, with a particular emphasis on the early requirements engineering phase.

In particular, Tropos aims at two important objectives:

1. raising the conceptual level of Requirements Engineering techniques, so that formal and semiformal languages and representations can be used since the very early stages of requirements elicitation and analysis (this means that empirical measures, tables, and transcripts or cards provided in free text form [23]) have to be transformed into more precise and more easily analyzable formats, so that transforming them into functional and non-functional requirements - to feed the Software Engineering process - results to be a more straightforward step);

2. providing and supporting the system architecture and functions definition with a set of "social-oriented" notions - to be used aside the traditional system oriented concepts - that allows for a easier mapping of the requirements provided in terms of social and organizational needs - as provided by Requirements Engineering - into the characteristics (functional, architectural, and design oriented) of the system-to-be.

Tropos aims at this objective by adopting two specific strategies:

1. It pays attention to the activities that precede the specification of the prescriptive requirements, like understanding how and why the intended system can meet the organizational goals (Late Requirements Analysis). Even before this phase, it is important to understand and analyze the organizational goals themselves (Early Requirements Analysis). In this, Tropos is largely inspired by the Eric Yu's $i^{*}$ framework for requirements engineering, which offers actors, goals, and actor dependencies as primitive concepts. The $i^{*}$ framework has been presented in detail in [29] and has been related to different application areas, including requirements engineering [28], business process reengineering [31], and software processes [30].

2. Tropos deals with all the phases of system requirement analysis and all the phases of system design and implementation in a uniform and homogeneous way, based on common mentalistic notions as those of actors, goals, softgoals, plans, resources, and intentional dependencies.

One of the main advantages of the Tropos methodology is that it allows to capture not only the what or the how, but also the why a piece of software is developed. This, in turn, allows for a more refined analysis of the system dependencies and, in particular, for a much better and uniform treatment not only of the system functional requirements, but also of its non-functional requirements.

The choice of focusing on the goals (and all the related mentalistic notions) along all the phases of the Tropos methodology, has in important impact on the overal process of software development, including the very implementation of the 
system, specially if, although not exclusively, an Agent Oriented Programming [18] is adopted. In particular, agent oriented specifications and programs use the same notions and abstractions used to describe the behavior of human agents and the processes involving them; thus, the conceptual gap between users' specifications (in terms of why and what) and system realization (in terms of what and how), is reduced to a minimum.

The Tropos methodology is mainly based on four phases [20,2]:

- Early Requirements Analysis, aimed at defining and understanding a problem by studying its existing organizational setting;

- Late Requirements Analysis, conceived to define and describe the system-tobe, in the context of its operational environment;

- Architectural Design, that deals with the definition of the system global architecture in terms of subsystems;

- Detailed Design, aimed at specifying each architectural component in further detail, in terms of inputs, outputs, control and other relevant information.

In particular, in this paper we will concentrate on the Early Requirements Analysis phase of Tropos. During the Early Requirements Analysis the existing organizational setting is analyzed, in terms of actors, who plays some role in the organization, and of their intentional dependencies in the context of the organization. The output of this phase is an organizational model which includes relevant actors and their respective intentional dependencies. Actors, in the organizational setting, are characterized by having goals that each single actor, in isolation, would be unable - or not as well or as easily - to achieve. Intentional dependencies are used to describe this kind of relationships among actors. Goals are the the elements around which the intentional dependencies are established.

Thus, in a nutshel we can say that the stated aim of Tropos is to use agent concepts in the description and definition of the methodology rather than using agent concepts in a minor extension to existing $\mathrm{OO}$ approaches. Tropos takes the BDI model $[22,18]$, formulated to describe the internal view of a single agent, and applies those concepts to the external view in terms of problem modelling as part of requirements engineering.

It is for this reason that, in Tropos, AI derived mentalistic notions such as actors (or agents), goals, soft-goals, plans, resources, and intentional dependencies are used in all the phases of software development, from the first phases of early analysis down to the actual implementation. Tropos also includes descriptions of Work Products and several Techniques such as Means-End Analysis, useful in requirements engineering.

A crucial role is given to the earlier analysis of requirements that precedes prescriptive requirements specification. In particular, aside from the understanding of how the intended system will fit into the organizational setting, and what the system requirements are, Tropos addresses also the analysis of the why the system requirements are as they are, by performing an in-depth justification with respect to the organizational goals. 


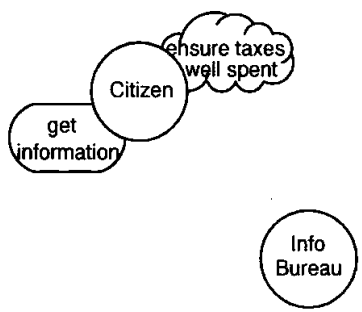

(a)

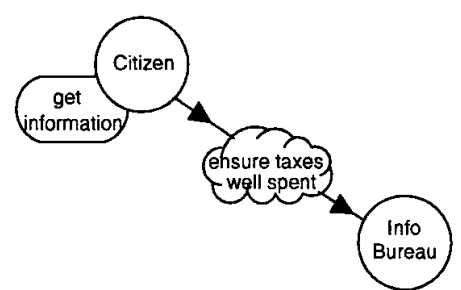

(b)

Fig. 5. (a) Example actor diagram showing goals attached to actors; (b) Example actor diagram showing an explicit dependee, depender and dependum

Thus, the stakeholder intentions are modelled as goals which, through a goaloriented analysis, eventually lead to the functional and non-functional requirements of the system-to-be. In Tropos, early requirements are assumed to involve social actors who depend on each other for goals to be achieved, tasks to be performed, and resources to be furnished. Tropos includes actor diagrams for describing the network of social dependency relationships among actors, as well as goal diagrams for analyzing goals through a means-ends analysis in order to discover ways of fulfilling them. These primitives have been formalized using intentional concepts from AI, such as goal, belief, ability, and commitment [2].

An Actor Diagram is a graph, where each node may represent either an actor, a goal, a soft-goal, a task or a resource. Links, among nodes, may be used to form paths like: depender $\rightarrow$ dependum $\rightarrow$ dependee, where the depender and the dependee are actors, and the dependum is either a goal, a soft-goal, a task or a resource. Each path between two actors indicates that one actor depends on another for something (represented by the dependum) so that the former may attain some goal/soft-goal/task/resource. In other terms, a dependency describes an "agreement" between two actors (the depender and the dependee), in order to attain the dependum. The depender is the depending actor, and the dependee the actor who is depended upon. The type of the dependum describes the nature of the dependency (and, therefore, of the implied agreement). Goal dependencies are used to represent delegation of responsibility for fulfilling a goal; soft-goal dependencies are similar to goal dependencies, but their fulfillment cannot be defined precisely (for instance, the appreciation is subjective, or the fulfillment can occur only to a given extent); task dependencies are used in situations where the dependee is required to perform a given activity; and resource dependencies require the dependee to provide a resource to the depender. As exemplified in Figure 5, actors are represented as circles; dependums - goals, soft-goals, tasks and resources - are respectively represented as ovals, clouds, hexagons and rectangles ${ }^{4}$.

\footnotetext{
${ }^{4}$ In this paper only examples of goals and soft-goals are shown.
} 


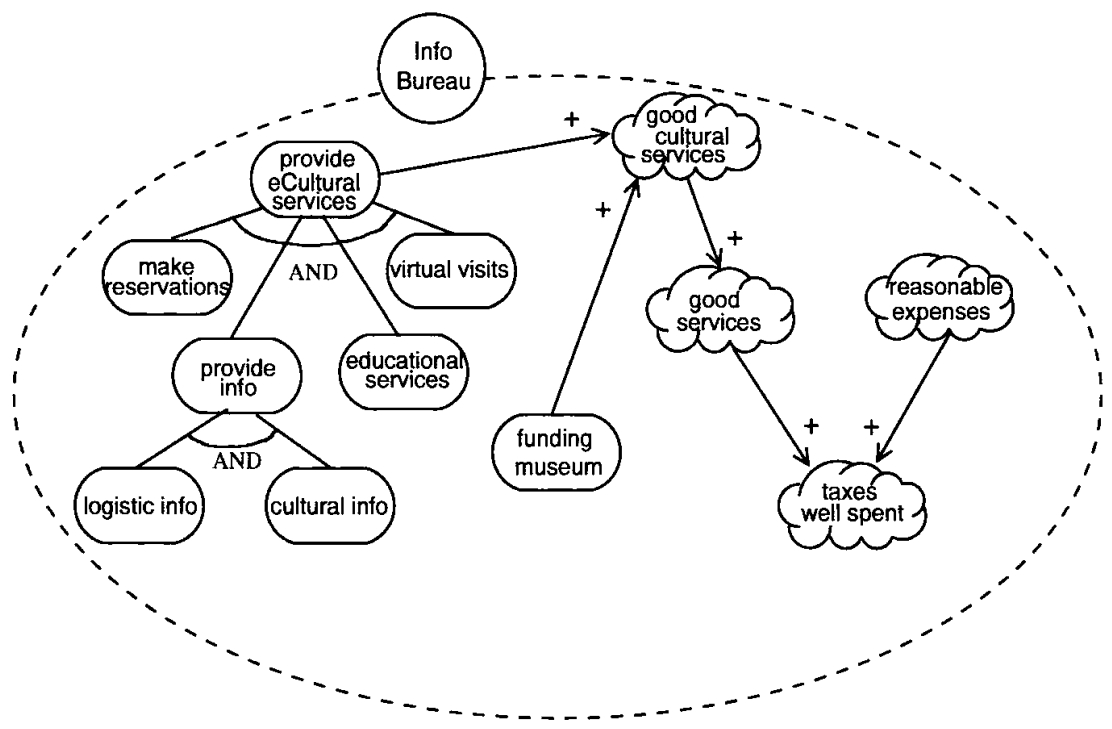

Fig. 6. Example goal diagram

As an example of actor diagram, let us consider Figure 5 (adapted from [2]), in which the following actors have been identified:

- Info Bureau, that is a government agency, the objectives of wich include improving public information services, increasing tourism through new information services, also encouraging Internet among the citizens.

- Citizens, who want easily accessible information, of any sort, and (of course) good administration of public resources.

Thus, in Figure 5-a the Citizen is characterized by one goal (get information) and one soft-goal (ensure taxes well spent). In Figure 5-b (that represent a first, simple evolution of figure Figure 5-a), the soft-goal (ensure taxes well spent is shown as a dependency between the Citizen and the Info Bureau.

Once the stakeholders have been identified, along with their goals and social dependencies, the analysis proceeds in order to enrich the model with further details. In particular, the rationale of each goal relative to the stakeholder who is responsible for its fulfillment has to be analyzed. Basically, this is done through means-end analysis and goal/plan decomposition. It is important to stress that what goals are associated with each actor is a decision of the corresponding stakeholder, not the design team.

An example of the result of such an analysis from the perspective of Info Bureau is given by the goal diagram depicted in Figure 6 . Here, the goal analysis for Info Bureau, relative to the goal that Citizen delegates to Info Bureau as a 


\begin{tabular}{||l|l||}
\hline Activity & \\
\hline Early requirements engineering & \\
\hline Tasks & Related Techniques \\
\hline Model actors & \\
\hline Model capabilities for actors & Capabilities identification and analysis \\
\hline Model dependencies for actors and goals & Delegation analysis \\
\hline Model goals & $\begin{array}{l}\text { Means-End Analysis } \\
\text { Contribution Analysis } \\
\text { AND/OR Decomposition }\end{array}$ \\
\hline Model plans & $\begin{array}{l}\text { Means-End Analysis } \\
\text { AND/OR Decomposition }\end{array}$ \\
\hline \hline Work Products & \\
\hline $\begin{array}{l}\text { (Tropos) actor diagram } \\
\text { (Tropos) capability diagram } \\
\text { (Tropos) goal diagram } \\
\text { (Tropos) plan diagram }\end{array}$ & \\
\hline
\end{tabular}

Table 2. Activity, Tasks, Techniques and Work Products proposed for inclusion in the OPF repository as a result of analyzing Tropos.

result of the previous analysis, is given. Inside the goal diagram, soft-goal analysis is performed identifying the goals and soft-goals that contribute positively (or negatively) to the soft-goal. For example, the soft-goal taxes well spent gets positive contributions from the soft-goal good services, and, in the end, from the goal provide eCulture services too.

The goal provide eCultural services is decomposed (AND decomposition) into four subgoals: make reservations, provide info, educational services and virtual visits. As basic eCultural service, the Info Bureau must provide information (provide info), which can be logistic info, and cultural info. More in detail, accordingly with the original scenario introduced in [2], Logistic info concerns, for instance, timetables and visiting instructions for museums, while cultural info concerns the cultural content of museums and special cultural events. This content may include descriptions and images of historical objects, the description of an exhibition, and the history of a particular region. Virtual visits are services that allow, for instance, Citizen to pay a virtual visit to a city of the past (Rome during Cæsar's time!). Educational services includes presentation of historical and cultural material at different levels (e.g., high school or undergraduate university level) as well as on-line evaluation of the student's grasp of this material. Make reservations allows the Citizen to make reservations for particular cultural events, such as concerts, exhibitions, and guided museum visits. 


\section{Supporting Tropos Concepts in the OPEN Process Framework}

In this section, we evaluate the existing Agent OPEN description (summarized in Section 2.2 above) against ideas formulated within the Tropos methodology, seeking any omissions or poor support of Tropos elements in the OPF. We then make recommendations for enhancements to the OPF in order that it can fully support all agent-oriented concepts formulated in Tropos.

Several new process components (method chunks) need to be added to the existing OPF repository. These are primarily Tasks and Techniques but there is also one new Activity: Early Requirements Engineering (in Tropos called the Early Requirements Analysis phase) as well as some work products. All of these are outlined below in standard OPEN format and summarized for convenience in Table 2.

\subsection{Activity}

An Activity in the OPF describes a coarse granular "job to be done". It describes "what" needs to be done but not "how". One new Activity is proposed here for inclusion in the OPF repository based on contributions made by Tropos.

Early Requirements Engineering Early requirements engineering focusses on domain modeling. It consists of identifying and analyzing the relevant actors in organizations and their goals or intentions. These actors may correspond with the stakeholders but may also include other social elements (individuals, but also organizations, organizational units, teams, and so on) who do not directly share an interest in the project, but still need to be modelled in order to produce a sufficiently complete picture of the organizational domain. Each organization active element is modelled as a (social) actor that is dependent upon another (social) actor in order for them to achieve some stated goal. During Early Requirements Engineering, these goals are decomposed incrementally and finally the atomic goals can be used to support an objective analysis of alternatives.

The results of this analysis can be documented using a variety of Tropos diagrams. Goals, actors and dependencies can be depicted on an actor diagram and, in more detail, on a goal diagram. These results then form the basis for the "late requirements analysis" which in OPEN is called simply Requirements Engineering in which the system requirements are elicited in the context of the stakeholders' goals identified in this activity of Early Requirements Engineering.

\subsection{Tasks}

A Task in the OPF describes a granular "job to be done". As with Activities, a Task describes what is to be done but not how. However, the granularity is at an individual developer's scale, in comparison to the team scale of an Activity. In this section, we describe five new/modified Tasks in the layout style used as standard in the OPEN literature (e.g. [11]). 


\section{Task: Model actors}

Focus: People, other systems and roles involved

Typical supportive techniques: Business process modelling, Soft systems analysis Explanation. While the concept of actors in $O O$ systems already exists (and is supported in the original OPF), the Tropos methodology extends the OO notion of an actor beyond that of a single person/system/role interacting with a system to that of a more general entity that has strategic goals and intentionality within the system or organizational setting [2] including also, for example, whole organizations, organizational units and teams. Actors in Tropos can represent either agents (both human and artificiai) or roles or positions (a set of roles, typically played by a single agent). This new Task thus considerably extends the existing concepts related to traditional $O O$ actors. To model an actor, one must identify and analyze actors of both the environment and the system (or system-to-be). Tropos encourages the use of this Task in the early requirements phase for the modelling of domain stakeholders and their intentions as social actors. Actors can be depicted using (Tropos) actor diagrams (see below).

\section{Task: Model capabilities for actors}

Focus: Capability of each actor in the system

Typical supportive techniques: Capabilities identification and analysis

Explanation. The capability of an actor represents its ability to define, choose and execute a plan (for the fulfilment of a goal), given specific external environmental conditions and a specific event [2]. Capability modelling commences after the architecture has been designed, subsequent to an understanding of the system sub-actors and their interdependencies. Each system subactor must be provided with its own individual capabilities, perhaps with additional "social capabilities" for managing its dependencies with other actors/subactors. Previously modelled goals and plans generally now become an integral part of the capabilities. Capabilities can be depicted using (Tropos) capability diagrams and plan diagrams (see below).

\section{Task: Model dependencies for actors and goals}

Focus: How/if an actor depends on another for goal achievements

Typical supportive techniques: Delegation analysis

Explanation. In Tropos, a dependency may exist between two actors so that one actor depends in some way on the other in order to achieve its own goal, a goal that cannot otherwise be achieved or not as well or as easily without involving this second actor. Similarly, a dependency between two actors may exist for plan execution or resource availability [2]. The actors are named, respectively, the depender and the dependee while the dependency itself centres around the dependum. Dependencies can be depicted using (Tropos) actor diagrams and, in more detail, in goal diagrams (see below).

\section{Task: Model goals}


Focus: Actor's strategic interests

Typical supportive techniques: Means-end analysis, contribution analysis, AND/OR decomposition

Explanation. A goal represents an actor's strategic interests [2] - Tropos recommends both hard and soft goals. Modelling goals requires the analysis of those actor goals from the view point of the actor itself. The rationale for each goal relative to the stakeholder needs to be analyzed - typical Techniques are shown in Table 2. Goals may be decomposed into subgoals, either as alternatives or as concurrent goals. Plans may also be shown together with their decomposition, although details of plans are shown in a Plan Diagram (q.v.). Goals can be depicted using (Tropos) goal diagrams (see below).

\section{Task: Model plans}

Focus: Means to achieve goals

Typical supportive techniques: Means-end analysis, AND/OR decomposition Explanation. A plan represents a means by which a goal can be satisfied or, in the case of a soft goal, satisficed [2,29]. Plan modelling complements goal modelling and rests on reasoning techniques analogous to those used in goal modelling. Plans can be depicted using (Tropos) goal diagrams and plan diagrams (see below).

\subsection{Techniques}

To complement the "what" of Activities and Tasks, OPF Techniques detail "how" they are to be achieved. We have identified four new Techniques from Tropos and describe them here in the standard format for OPF Techniques [17].

\section{Technique: Means-End Analysis}

Focus: Identifying means to achieve goals

Typical tasks for which this is needed: Model goals, Model plans

Description. Means-end analysis aims at identifying plans, resources and goals as well as means to achieve the goals.

Usage. To perform means-end analysis, the following are performed iteratively:

- Describe the current state, the desired state (the goal) and the difference between the two

- Select a promising procedure for enabling this change of state by using this identified difference between present and desired states.

- Apply the selected procedure and update the current state.

If this successfully finds an acceptable solution, then the iterations cease; otherwise they continue. If no acceptable solution is possible, then failure is announced. 


\section{Technique: Contribution Analysis}

Focus: Goals contributing to other goals

Typical tasks for which this is needed: Model goals

Description. Contribution analysis identifies goals that may contribute to the (partial) fulfilment of the final goal. It may be alternatively viewed as a kind of means-end analysis in which the goal is identified as the means [2]. Contribution analysis applied to soft-goals is often used to evaluate non-functional requirements.

Usage. Identify goals and soft-goals that can contribute either positively or negatively towards the achievement of the overall goal or soft-goal. Of course the focus is on identifying positive contributions, but the technique may also lead, as a side effect, to the identification of negative contributions. Annotate these appropriately (say with + or - ). A + label indicates a positive, partial contribution to the fulfilment of the goal being analyzed. Contribution analysis is very effective for soft goals used for eliciting non-functional (quality) requirements.

\section{Technique: AND/OR Decomposition}

Focus: Goal decomposition

Typical tasks for which this is needed: Model goals, Model plans

Description. This is a technique to decompose a root goal into a finer goal structure.

Usage. Start with a high level goal and decompose into subgoals. These subgoals may either be alternatives (OR decomposition) or additive (AND decomposition).

\section{Technique: Capabilities identification and analysis}

Focus: Capabilities identification

Typical tasks for which this is needed: Model capabilities for actors

Description. For each goal introduced, we identify a set of capabilities that the responsible actor should have in order to fulfill the goal. When the achievement of the goal involves other actors, the analysis is expanded also to these actors. Capabilities for the interaction/collaboration are then identified and analyzed contextually (see [2] for more details).

Usage. Start with a goal associated to an actor and identify the capabilities needed locally. If the goal involves other actors the analysis is extended to these actors with respect to their contribution in the achievement of the goal.

Finally, we had to consider also the technique Delegation Analysis. Indeed, this technique is not new in OPF (see [17]), but its original focus is on modeling objects, possibly to create components, and it is aimed at transforming designs. In the Tropos context, instead, Delegation Analysis processes the (Tropos) Actor Diagrams, that are work products of both the Early Requirements Engineering activity ("early requirements analysis" in Tropos) and the Requirements Engineering activity ("late requirements analysis" in Tropos). An example 
of (Tropos) Delegation Analysis is presented in Section 4.4, as the transformation of Figure 5-a into Figure 5-b. Here, we simply recommend modification of the Delegation Analysis technique introduced in [17], in order to deal also with the agent and Tropos typical notions (i.e., actor, goal, task, resource, depender, dependum and dependee), so as to fully accommodate the Tropos process.

\subsection{Work Products}

OPF Work Products describe artefacts that are created, consumed and/or maintained. Some of these act as deliverables, either to other team members or to a third party client. Four new work products are identified and described here.

Work Product: (Tropos) Actor Diagram In Tropos, the actor diagram graphically depicts actors (as circles), their goals (as ellipses and clouds) attached to the relevant actor (Figure 5-a) together with a network of dependencies between the actors (Figure 5-b). In Figure 5-a, Citizen has two goals: the hard goal to get information and the soft goal to ensure taxes well spent. However, this soft goal is best delegated to the Info Bureau actor. To show this delegation, the delegated goal is shown explicitly as a dependum (cloud symbol) connected by two line segments to the two actors (Citizen and Info Bureau) (Figure 5-b).

Work Product: (Tropos) Capability Diagram A capability diagram is drawn from the viewpoint of a specific agent. They are initiated by an event caused by an external event. Nodes in the diagram model plans (which can be expanded through the use of a Plan Diagram (q.v)) and transition arcs model events. Beliefs are modelled as objects [2]. Each node in the capability diagram may be expanded into a Plan Diagram (q.v.). Capability diagrams in Tropos use UML activity diagrams.

Work Product: (Tropos) Goal Diagram Figure 6 shows an example goal diagram in which the focus is that of how Info Bureau tries to achieve the delegated soft-goal taxes well spent. Providing good services with reasonable expenses, Info Bureau can contribute to spend taxes well. Good services may include good cultural services, which in turn may include services available via the web. So provide eCultural services can contribute positively in achieving the sotfgoal good cultural services. Figure 6 shows also the partial AND decomposition of the provide eCultural services goal.

Work Product: (Tropos) Plan Diagram A plan diagram depicts the internal structure of a plan, summarized as a single node on a Capability diagram (q.v.). Plan diagrams in Tropos use UML activity diagrams. 


\section{Conclusions and Future Work}

Based on an understanding that $\mathrm{OO}$ methodologies can usefully be enhanced to support agency, we report here on the first results of a project to extend the OPEN Process Framework (OPF) to include agent-oriented support found in various agent-oriented (AO) methodologies. Initial analysis of the Tropos $A O$ methodology identifies its significant support for early requirements. It captures many aspects of agent-oriented requirements gathering not previously documented.

In analyzing the extent to which other methodological frameworks, and in particular the OPEN Process Framework, supports these ideas, many deficiencies were identified. Here, we have itemized these gaps in OPEN's repository of process components and proposed additions to the repository specifically to address activities, tasks, techniques and work products found in Tropos but, until now, not available in the OPF repository.

We intend to progress this cross-fertilization between OPEN and Tropos, specifically taking advantage of the strengths of each: the early requirements engineering and agent focus of Tropos and the full lifecycle process of OPEN together with its metamodel-based underpinning that permits it to be used for situated method engineering [3].

\section{References}

1. Bernon, C., Gleizes, P.-P., Picard, G. and Glize, P., The ADELFE methodology for an intranet system design, Procs. Agent-Oriented Information Systems 2002 (eds. P. Giorgini, Y. Lespérance, G. Wagner and E. Yu), May 2002, Toronto, Canada.

2. Bresciani, P., Giorgini, P., Giunchiglia, F., Mylopolous, J. and Perini, A.: Tropos: an agent-oriented software development methodology. Journal of Autonomous MultiAgent Systems (2003) in press

3. Brinkkemper, S.: Method engineering: engineering of information systems development methods and tools. Inf. Software Technol. 38(4) (1996) 275-280

4. Caire, G., Chainho, P., Evans, R., Garijo, F., Gomez Sanz, J., Kearney, P., Leal, F., Massonet, P., Pavon, J. and Stark, J., Agent-oriented analysis using MESSAGE/UML, Procs. Second Int. Workshop on Agent-Oriented Software Engineering (AOSE-2001), Montreal, Canada, May 2001, 101-107 (2001)

5. Castro J., Kolp M. and Mylopoulos J.: Towards Requirements-Driven Information Systems Engineering: The Tropos Project. Information Systems. Elsevier, Amsterdam, the Netherlands (2003) in press

6. Debenham, J. and Henderson-Sellers, B., Designing agent-based process systems - extending the OPEN Process Framework, Chapter VIII in Intelligent Agent Software Engineering (ed. V. Plekhanova), Idea Group Publishing (2003) 160-190

7. DeLoach, S.A., Multiagent systems engineering: a methodology and language for designing agent systems, Procs. Agent-Oriented Information Systems '99 (AOIS'99), Seattle, WA, USA, 1 May (1999)

8. Duncan, W.R.: A Guide to the Project Management Body of Knowledge, Project Management Institute, PA, USA (1996) $176 \mathrm{pp}$

9. Firesmith, D.G. and Henderson-Sellers, B.: The OPEN Process Framework. An Introduction, Addison-Wesley, Harlow, UK (2002) 330pp 
10. Giorgini P., Perini A., Mylopoulos J., Giunchiglia F. and Bresciani P.: AgentOriented Software Development: A Case Study . Proceedings of the Thirteenth International Conference on Software Engineering and Knowledge Engineering (SEKE01), June 13-15 2001, Buenos Aires, Argentina (2001)

11. Graham, I., Henderson-Sellers, B. and Younessi, H.: The OPEN Process Specification, Addison-Wesley, Harlow, UK (1997) 314pp

12. Haire, B., Henderson-Sellers, B. and Lowe, D., Supporting web development in the OPEN process: additional tasks, Procs. 25th Annual International Computer Software and Applications Conference. COMPSAC 2001, IEEE Computer Society Press, Los Alamitos, CA, USA (2001) 383-389

13. Haire, B., Lowe, D. and Henderson-Sellers, B., Supporting web development in the OPEN process, Object-Oriented Information Systems (eds. Z. Bellahsène, D. Patel and C. Rolland), LNCS 2425, Springer-Verlag, 2002.

14. Henderson-Sellers, B., An OPEN process for component-based development, Chapter 18 in Component-Based Software Engineering: Putting the Pieces Together (eds. G.T. Heineman and W. Councill), Addison-Wesley, Reading, MA, USA, 2001.

15. Henderson-Sellers, B. and Debenham, J., 2003, Towards OPEN methodological support for agent oriented systems development, Procs. First International Conference on Agent-Based Technologies and Systems (eds. B.H. Far, S. Rochefort and M. Moussavi), University of Calgary, Calgary, Canada, 14-24

16. Henderson-Sellers, B. and Serour, M., Creating a process for transitioning to object technology, Proceedings Seventh Asia-Pacific Software Engineering Conference. APSEC 2000, IEEE Computer Society Press, Los Alamitos, CA, USA, 2000.

17. Henderson-Sellers, B., Simons, A.J.H. and Younessi, H.: The OPEN Toolbox of Techniques, Addison-Wesley, UK (1998) 426pp + CD

18. Kinny, D., Georgeff, M. and Rao, A., A methodology and modelling techniques for systems of BDI agents, TR 58, Australian Artificial Intelligence Institute (1996)

19. OMG: OMG Unified Modeling Language Specification, Version 1.4, September 2001, OMG document formal/01-09-68 through 80 (13 documents) [Online]. Available http://www.omg.org (2001)

20. Perini A., Bresciani P., Giorgini P., Giunchiglia G. and Mylopoulos J.: A Knowledge Level Software Engineering Methodology for Agent Oriented Programming. In J. P. Müller, E. Andre, S. Sen, and C. Frasson, editors, Proceedings of the Fifth International Conference on Autonomous Agents, May 2001, Montreal, Canada, 2001.

21. Perini A., Bresciani P., Giorgini P., Giunchiglia F. and Mylopoulos J.: Towards an Agent Oriented approach to Software Engineering. In A. Omicini and M. Viroli, editors, WOA 2001 - Dagli oggetti agli agenti: tendenze evolutive dei sistemi software, 4-5 September 2001, Modena, Italy, Pitagora Editrice Bologna (2001)

22. Rao, A.S. and Georgeff, M.P., BDI agents: from theory to practice, Technical Note 56, Australian Artificial Intelligence Institute (1995)

23. Robertson, S. and Robertson, J., Mastering the requirements process. Number 0201360462 in ACM press books. Addison-Wesley (1999)

24. Rupprecht, C., Fünffinger, M., Knublauch, H. and Rose, T., Capture and dissemiantion of experience about the construction of engineering processes, Procs. CAiSE 2000, LNCS 1789, Springer Verlag, Berlin, 294-308 (2000)

25. Ter Hofstede, A.H.M. and Verhoef, T.F., On the feasibility of situational method engineering, Information Systems, 22, 401-422 (1997)

26. van Slooten, K., Hodes, B., Characterizing IS development projects, in Proceedings of the IFIP TC8 Working Conference on Method Engineering: Principles of method construction and tool support (eds. S. Brinkkemper, K. Lyytinen, R. Welke) Chapman\&Hall, Great Britain, 29-44 (1996) 
27. Wooldridge, M., Jennings, N.R. and Kinny, D., The Gaia methodology for agentoriented analysis and design, J. Autonomous Agents and Multi-Agent Systems, 3, 285-313 (2000)

28. E. Yu. Modeling organizations for information systems requirements engineering. In Proceedings of the First IEEE International Symposium on Requirements Engineering, pages 34-41, San Jose, January 1993. IEEE.

29. E. Yu. Modelling Strategic Relationships for Process Reengineering. PhD thesis, University of Toronto, Department of Computer Science, 1995.

30. E. Yu and J. Mylopoulos. Understanding 'why' in software process modeling, analysis and design. In Proceedings Sixteenth International Conference on Software Engineering, Sorrento, Italy, May 1994.

31. E. Yu and J. Mylopoulos. Using goals, rules, and methods to support reasoning in business process reengineering. International Journal of Intelligent Systems in Accounting, Finance and Management, 1(5), January 1996. 